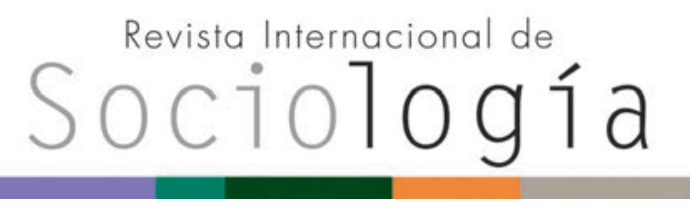

Revista Internacional de Sociología RIS vol. 76 (3), e106, julio-septiembre, 2018, ISSN-L:0034-9712 https://doi.org/10.3989/ris.2018.76.3.17.60

\section{GÉNERO Y COMMUTING EN LAS REGIONES URBANAS ANDALUZAS}

\author{
José Manuel ToRrado \\ Universidad de Granada \\ josetr@ugr.es
}

ORCID iD: https://orcid.org/0000-0003-2597-4699

\author{
JAVIER Romaní \\ Universidad de Barcelona \\ romani@ub.edu \\ ORCID iD: https://orcid.org/0000-0001-7998-1056
}

JOAQUÍN SUSINO

Universidad de Granada

jsusino@ugr.es

ORCID iD: https://orcid.org/0000-0001-9096-6172

Cómo citar este artículo / Citation: Torrado, J. M., J. Romaní y J. Susino 2018. "Género y commuting en las regiones urbanas andaluzas". Revista Internacional de Sociología 76(3): e106. https://doi.org/10.3989/ ris.2018.76.3.17.60

\footnotetext{
Resumen

Las diferencias entre mujeres y hombres en las distancias recorridas en los desplazamientos residencia-trabajo son un objeto recurrente en la literatura sobre movilidad, al constatar que las mujeres trabajan más cerca de casa. Sin embargo, el modo en el que estas diferencias operan es objeto de debate entre dos perspectivas: la teoría del enclaustramiento femenino y la teoría tradicional. La primera afirma que las diferencias entre sexos en la movilidad se deben al género, en concreto, a la mayor carga de trabajo doméstico de las mujeres y a la relegación de su rol laboral. Mientras la segunda hace énfasis en cómo estas diferencias se deben a factores intermedios, principalmente socioeconómicos. Nuestro objetivo consiste en contrastar ambas hipótesis para el caso andaluz, a través de la Encuesta Social 2011 de Movilidad en las Regiones Urbanas de Andalucía. Los resultados obtenidos apuntan a la complementariedad de las mismas, aunque operando las desigualdades de género más de manera indirecta, por vía segregación ocupacional, que directa.
}

\section{Palabras Clave}

Desigualdad de género; Distancia; Enclaustramiento femenino; Movilidad por trabajo; Segregación ocupacional.
Copyright: ( 2018 CSIC. Este es un artículo de acceso abierto distribuido bajo los términos de la licencia de uso y distribución Creative Commons Reconocimiento 4.0 Internacional (CC BY 4.0).

Recibido: 20/04/2017. Aceptado: 15/03/2018

\section{Abstract}

Sex differences in commuting have been a recurring object since finding that women work closer to home. However, the way in which they operate is the subject of debate between two perspectives: the female entrapment theory and the traditional theory. The first one tells us that sex differences in commuting are due to gender, in particular to the greater domestic workload and the relegation of the female labor role as secondary. While the second emphasizes how these differences are due to intermediate factors, mainly socio-economics. The objective of the present study is to contrast both hypotheses for the Andalusian case, through the 2011 Social Survey of mobility in Andalusian urban regions. The results obtained point to the complementarity of both, finding that gender inequalities operate more indirectly, through occupational segregation, than directly.

\section{KeYWORDS}

Distance; Female entrapment; Gender inequality; Occupational segregation; Travel-to-work. 


\section{INTRODUCCIÓN}

Con una población de 8,4 millones habitantes, en Andalucía hay un total de 9 regiones urbanas, que concentran cerca del $60 \%$ de su población (IECA 2011), aunque son regiones de diferente dimensión y con fenómenos metropolitanos de distinta naturaleza (Feria 2015). Una diversidad que puede ser considerada representativa de la totalidad del universo metropolitano español que, a excepción de los casos de Madrid y Barcelona, presenta ciertas características peculiares que lo diferencian de la realidad territorial del norte de Europa, debido a su relativo retraso histórico en términos económicos y de movilidad (Cheshire 1995).

En 2011, la movilidad por trabajo en Andalucía presentaba unos tiempos medios de desplazamiento que rondaban los veinticuatro minutos, predominando los recorridos de distancias inferiores a 3 kilómetros, que podríamos llamar de proximidad, en el 47 $\%$ de los desplazamientos; siendo el vehículo privado a motor el medio por el que se realizaron más de las tres cuartas partes de dichos desplazamientos (IECA 2011). Estos valores agregados difieren de los registrados en las dos principales regiones urbanas españolas: Madrid y Barcelona (RodríguezMoya y García-Palomares 2012; Miralles-Guasch y Melo 2012), así como en el contexto internacional (McKenzie y Rapino 2011). Destacan el menor tiempo medio de desplazamiento y el menor uso del transporte público en el caso andaluz, que hacen de Andalucía una realidad territorial y social diferente de aquellas en las que se han realizado la mayoría de estudios sobre commuting.

Denominaremos commuting a la movilidad cotidiana por razón de trabajo, siguiendo la literatura internacional, en la que se suele emplear en sentido amplio, independientemente del ámbito territorial en que los movimientos residencia-trabajo se produzcan (Preston y McLafferty 2016; Sang, O'Kelly y Kwan 2011; Crane 2007, entre otros); aunque también en sentido restringido, referida a los movimientos entre unidades administrativas diferentes (Kim et al. 2012; Artís, Romaní y Suriñach 2000; por ejemplo). El motivo por el que nos centramos en este tipo de movilidad es doble. En primer lugar, porque es la forma de movilidad que de manera más decisiva estructura espacialmente las áreas urbanas funcionales, hasta el punto de que, desde hace años, allí donde los datos han estado disponibles, es la principal variable considerada en la delimitación de áreas metropolitanas (Feria 2004). En segundo lugar, porque el commuting afecta a mujeres y hombres en una gran proporción, estando menos confinado a grupos de edad específicos que la movilidad por estudios y caracterizándose por pautas cotidianas recurrentes y duraderas, en mucha mayor medida que otras motivaciones, como las compras o el ocio. Por ello, el commuting es un tipo de movilidad especialmente interesante para analizar las diferencias de género, menos evidentes que en otros casos en que se derivan de la propia desigualdad de los roles de género en la vida cotidiana, como ocurre con las compras o las visitas a familiares.

La literatura sobre los determinantes de la movilidad laboral ha identificado una amplia gama de factores directamente relacionados con la distancia, los tiempos y los modos de transporte utilizados. Estos factores determinantes podrían clasificarse en tres bloques principales de variables o vectores, que guardan estrechas relaciones entre sí (Artís, Romaní y Suriñach 2000). En primer lugar, destacan los factores territoriales, referidos al lugar donde se reside y trabaja, tanto por lo que se refiere a la región urbana en que se habita como a la diferencia entre cabecera o zona suburbana, siempre en interacción con otras variables (Preston y McLafferty 2016). En segundo lugar, las características socioeconómicas o profesionales de los commuters, referidas a los ingresos y a otras características ligadas a la ocupación. Y, por último, las sociodemográficas, entre las que el género (en tanto que sistema estructurado de desigualdad entre sexos) es una variable crucial, por cuanto supone diferencias sustanciales en las identidades, prácticas cotidianas, acceso al mercado laboral y, cómo no, en el uso del espacio por hombres y mujeres. La constatación de tales diferencias ha generado todo un tópico, tanto en la literatura española (Olmo y Maeso 2013; García-Palomares 2008; Díaz-Muñoz y Jiménez-Gigante 2007; Casado 2000; Díaz-Muñoz 1989, entre otros) como internacional (Dargay y Clark 2012; Crane 2007; Madden 1981, entre otros). En este contexto se encuadra el presente trabajo, cuya principal novedad reside en su estrategia de análisis, que pretende, más allá de constatar las desigualdades de género en la movilidad, ahondar en el conocimiento de los factores a través de los cuales dichas desigualdades operan, separando los directos (propiamente de género) de los indirectos (relacionados con la segregación ocupacional).

\section{Commuting y Desigualdades DE GÉNERO: LA TEORÍA DEL ENCLAUSTRAMIENTO Y LA TEORÍA TRADICIONAL}

Revisando las principales aportaciones referentes al commuting y su relación con las desigualdades de género, encontramos un gran consenso en la literatura en torno a la idea de que las mujeres trabajan más cerca de casa. Esta constatación ha servido como punto de partida para la mayoría de estudios que han tratado de analizar cómo el género puede incidir en la movilidad.

Este consenso se manifiesta de manera ilustrativa en el título del ya clásico trabajo de Madden (1981): Why women work closer to home? Y es que, en lo que respecta a las distancias recorridas, las mujeres 
tienden a trabajar más cerca del ámbito doméstico, hecho contrastado en contextos sociales, geográficos e históricos tan diferentes como Seúl (Lee y McDonald 2003), Sao Paolo (Neto, Duarte y Páez 2014), New York (McLafferty y Preston 1997; Preston y McLafferty, 2016) o Irlanda del Norte (Moss, Jack y Wallace 2004), por poner sólo algunos ejemplos. Incluso en la región objeto de estudio (Andalucía) un trabajo colectivo coordinado por Feria y Susino (2005) constató que, en efecto, los mercados locales de trabajo femeninos eran espacialmente más reducidos que los masculinos.

Sin embargo, aunque parezca un hecho casi universal que las mujeres trabajan más cerca de casa y que lo hacen por las desigualdades de género, parece no existir un consenso en torno a cómo actúan dichas desigualdades.

Ya Rosenbloom y Burns (1993) establecían dos perspectivas al respecto. La primera era identificada como la teoría económica tradicional (Gordon, Kumar y Richardson 1989; Hanson y Johnston 1985;), la cual afirmaba que las diferencias de sexo encontradas en la movilidad laboral se debían principalmente a la desigualdad de género en el mercado de trabajo, desigualdad que se manifestaba en los menores salarios de las mujeres, su mayor precariedad laboral $\mathrm{y}$, sobre todo, en su concentración en determinadas ocupaciones. Esta perspectiva es criticada parcialmente por las autoras citadas, las cuales parecían bosquejar, ya en los años noventa, la hipótesis complementaria, que más tarde fue bautizada como del enclaustramiento femenino (Sandow 2008; Crane 2007; Cristaldi 2005; Lee y McDonald 2003;). Esta hipótesis hace hincapié en que las diferencias de sexo en la movilidad se deben a efectos propios de la socialización diferencial entre hombres y mujeres, que hacen que éstas estén más interesadas en trabajar más cerca de casa, debido a su rol cuidador y a la percepción de su rol laboral como algo secundario.

Quedaba, pues, inaugurado un debate tácito entre dos perspectivas en apariencia contradictorias, a las que a partir de aquí nos referiremos como teoría tradicional y teoría del enclaustramiento femenino. La principal discrepancia entre ambas reside en que la primera afirma que las desigualdades de género operan de manera indirecta, a través de variables principalmente socioeconómicas (Hanson y Prat 1994), vinculadas a la segregación por sexo en la estructura ocupacional y sectorial; mientras la segunda parece mostrar que dichas desigualdades operan también de manera directa en la movilidad misma, no siendo la movilidad un mero epifenómeno de la segregación laboral por sexo, sino una manifestación más de la desigualdad entre hombres y mujeres (Olmo y Maeso 2013).

Así, algunos autores se han centrado en la explicación de las diferencias de sexo a través de la teoría del enclaustramiento, haciendo hincapié en cómo el estatus matrimonial y las cargas familiares son las principales causas de que comiencen a operar dichas diferencias en la movilidad por trabajo (Neto, Duarte y Páez 2014; Rapino y Cook 2011; Kawase 2004). Pero estudios recientes en el ámbito nacional e internacional han tendido a relajar este debate, mostrando su complementariedad, incluida la propia Rosenbloom (2006). Estos trabajos tienden a integrar ambas hipótesis, introduciendo en el análisis variables socioeconómicas y sociodemográficas que muestran que el efecto de género puede ser tanto directo como indirecto, en la medida en que puede actuar a través de otras variables mediadoras y operar de manera diferente para distintos grupos (Preston y McLafferty 2016) y en distintos territorios (Sang, O'Kelly y Kwan 2011). Es, por tanto, necesario aislar los efectos de la ocupación y el género y analizar las diferencias entre hombres y mujeres, controlando a su vez por otras variables socio-económicas, demográficas y territoriales distintas de éstas (Kim et al. 2012; McQuaid y Chen 2012; García-Palomares, 2008; Moss, Jack y Wallace 2004). Así, aunque es cierto que las diferencias de género se manifiestan en la movilidad de manera directa, "esta explicación ha sido siempre parcial porque esto no da cuenta de los largos desplazamientos de las mujeres con mayores niveles de formación en ocupaciones profesionales" (Hanson y Prat 1994: 500) y "tiene poca relevancia para muchas mujeres pertenecientes a minorías étnicas" (Preston y McLafferty 2016: 2).

Por eso, pese al intento reciente de conciliar ambas posturas, poco consenso existe en torno a la mayor o menor importancia de los distintos factores en la explicación de las diferencias de sexo en el commuting. Es decir, no hay acuerdo acerca de qué peso tiene el género en la explicación del commuting respecto a otras variables explicativas.

En el caso de Andalucía, existen también evidencias que apuntan a la confirmación de ambas hipótesis. Aunque una publicación reciente (Olmo y Maeso 2013) afirma que las diferencias de sexo en la movilidad son debidas precisamente a las desigualdades de género que operan sobre ellas, nuestra postura es que, aunque esto pueda ser parcialmente cierto, a dichas conclusiones sólo puede llegarse si se estudian las pautas por sexo de manera agregada, sin tener en cuenta la heterogeneidad interna de grupos sociales y ocupacionales. Otros estudios anteriores han demostrado que, para la misma ocupación y sectores de actividad, las pautas de movilidad por sexo pueden variar realmente poco (Feria y Susino 2005), apuntando directamente a la que Rosenbloom y Burns llamaron teoría tradicional como principal marco explicativo de dichas diferencias, sin que ello suponga negar que una parte de dichas diferencias puedan ser explicadas directamente por el género. 


\section{Objetivos, datos y metodología}

Como hemos visto, las diferencias entre sexos en la movilidad por trabajo y el efecto del género sobre las mismas, pese a ser un tópico extendido en la literatura internacional, no han sido muy tratadas en el caso andaluz, ámbito de nuestro estudio, debido principalmente a la carencia, hasta 2011, de bases de datos que recogieran información sobre desplazamientos cotidianos, y más concretamente, a la inexistencia de datos acerca de la distancia recorrida.

Ante el relativo vacío empírico y considerando el debate implícito existente entre las dos hipótesis complementarias (la teoría económica tradicional y la teoría del enclaustramiento), en este trabajo nos centramos en contrastarlas. Así, partiendo de un interrogante principal ( $¿ a$ través de qué factores operan las desigualdades de género en el commuting?), hemos definido dos objetivos principales.

El primer objetivo es constatar las diferencias de sexo existentes en las distancias recorridas (nuestra variable dependiente), separando hombres y mujeres, para cada una de las categorías de las variables independientes incluidas en el análisis, haciendo especial hincapié en las referentes a la ocupación. Se busca clasificar estas categorías en función de dichas diferencias de sexo, según su parecido o divergencia con respecto a la pauta agregada, estable- ciendo categorías con marcadas pautas de género frente a otras con pautas de movilidad propias.

El segundo objetivo se dirige a contrastar ambas hipótesis, a través de la realización de un modelo de regresión logística multinomial, que nos permitirá cuantificar el efecto del género y el resto de variables independientes (ver cuadro 1) a través de los coeficientes de regresión, así como el cambio que producen en el estadístico de bondad de ajuste. Los datos empleados corresponden a la Encuesta Social 2011 de Movilidad en las Regiones Urbanas de Andalucía (IECA 2011). Esta encuesta consta de 6 bloques, de los cuales hemos elegido el bloque 2 , que recoge, por primera vez para Andalucía, datos referentes a los desplazamientos de los trabajadores, con una amplia gama de variables sobre los individuos y sobre los desplazamientos mismos. Fueron encuestados 5767 hogares, en los cuales residían 2301 ocupados que realizaron un total de 2389 desplazamientos, poco más de uno por ocupado. Se han eliminado de la muestra un total de diez casos, correspondientes a desplazamientos de alumnos en prácticas, dado que para ellos no figuran variables centrales para nuestro trabajo, como la ocupación o el sector de actividad. Por tanto, la muestra final es de 2379 desplazamientos. Las variables utilizadas, tanto en el descriptivo como en el modelo, se recogen en el cuadro 1.

\section{Cuadro 1.}

Estadísticos descriptivos de las variables empleadas

\begin{tabular}{|c|c|c|c|c|c|c|}
\hline & \multicolumn{2}{|c|}{ Hombre } & \multicolumn{2}{|c|}{ Mujer } & \multicolumn{2}{|c|}{ Total } \\
\hline & Media & $\begin{array}{r}\text { Desviación } \\
\text { típica }\end{array}$ & Media & $\begin{array}{r}\text { Desviación } \\
\text { típica }\end{array}$ & Media & $\begin{array}{r}\text { Desviación } \\
\text { típica }\end{array}$ \\
\hline Edad & 41,68 & 9,95 & 40,51 & 10,08 & 41,17 & 10,02 \\
\hline \multirow[t]{2}{*}{ Edad al cuadrado } & 1836,01 & 843,99 & 1742,19 & 847,73 & 1795,39 & 846,71 \\
\hline & Frecuencia & $\begin{array}{r}\% \text { por } \\
\text { categoría }\end{array}$ & Frecuencia & $\begin{array}{r}\% \text { por } \\
\text { categoría }\end{array}$ & Frecuencia & $\begin{array}{r}\% \text { respecto } \\
\text { al total }\end{array}$ \\
\hline \multicolumn{7}{|c|}{ Variable dependiente } \\
\hline \multicolumn{7}{|c|}{ Distancia de desplazamiento: } \\
\hline 0 a $1 \mathrm{~km}$ & 244 & $46 \%$ & 283 & $54 \%$ & 527 & $22 \%$ \\
\hline 1 a $3 \mathrm{~km}$ & 334 & $55 \%$ & 276 & $45 \%$ & 610 & $26 \%$ \\
\hline 3 a $8,5 \mathrm{~km}$ & 368 & $59 \%$ & 252 & $41 \%$ & 620 & $26 \%$ \\
\hline 8,5 a $18 \mathrm{~km}$ & 214 & $60 \%$ & 144 & $40 \%$ & 358 & $15 \%$ \\
\hline más de 18 km & 174 & $72 \%$ & 67 & $28 \%$ & 241 & $10 \%$ \\
\hline \multicolumn{7}{|c|}{ Variables independientes } \\
\hline \multicolumn{7}{|l|}{ Estado civil: } \\
\hline Soltero & 331 & $51 \%$ & 316 & $49 \%$ & 647 & $27 \%$ \\
\hline Casado & 925 & $61 \%$ & 590 & $39 \%$ & 1515 & $64 \%$ \\
\hline Estuvo casado & 93 & $43 \%$ & 124 & $57 \%$ & 217 & $9 \%$ \\
\hline \multicolumn{7}{|l|}{ Nacionalidad: } \\
\hline Español & 1297 & $57 \%$ & 984 & $43 \%$ & 2281 & $96 \%$ \\
\hline Extranjero & 52 & $53 \%$ & 46 & $47 \%$ & 98 & $4 \%$ \\
\hline \multicolumn{7}{|l|}{ Estudios: } \\
\hline Sin estudios & 27 & $50 \%$ & 27 & $50 \%$ & 54 & $2 \%$ \\
\hline
\end{tabular}




\begin{tabular}{|c|c|c|c|c|c|c|}
\hline Primarios & 96 & $66 \%$ & 49 & $34 \%$ & 145 & $6 \%$ \\
\hline Secundarios & 437 & $64 \%$ & 251 & $36 \%$ & 688 & $29 \%$ \\
\hline Bachiller/Formación profesional & 428 & $58 \%$ & 304 & $42 \%$ & 732 & $31 \%$ \\
\hline Superiores & 361 & $48 \%$ & 399 & $53 \%$ & 760 & $32 \%$ \\
\hline \multicolumn{7}{|l|}{ Situación profesional: } \\
\hline Empresarios y autónomos & 379 & $72 \%$ & 147 & $28 \%$ & 526 & $22 \%$ \\
\hline Asalariado indefinido & 744 & $55 \%$ & 598 & $45 \%$ & 1342 & $56 \%$ \\
\hline Asalariado temporal & 185 & $44 \%$ & 231 & $56 \%$ & 416 & $17 \%$ \\
\hline Otra & 41 & $43 \%$ & 54 & $57 \%$ & 95 & $4 \%$ \\
\hline \multicolumn{7}{|l|}{ Tipo de contrato: } \\
\hline Tiempo completo & 1271 & $62 \%$ & 763 & $38 \%$ & 2034 & $85 \%$ \\
\hline Tiempo parcial & 78 & $23 \%$ & 267 & $77 \%$ & 345 & $15 \%$ \\
\hline \multicolumn{7}{|l|}{ Ocupación: } \\
\hline Directivos & 197 & $82 \%$ & 44 & $18 \%$ & 241 & $10 \%$ \\
\hline Profesionales y técnicos de salud y enseñanza & 99 & $35 \%$ & 185 & $65 \%$ & 284 & $12 \%$ \\
\hline Resto de profesionales y técnicos & 299 & $64 \%$ & 170 & $36 \%$ & 469 & $20 \%$ \\
\hline Empleados de oficina & 110 & $38 \%$ & 178 & $62 \%$ & 288 & $12 \%$ \\
\hline Trabajadores de servicios de cara al público & 202 & $34 \%$ & 391 & $66 \%$ & 593 & $25 \%$ \\
\hline Trabajadores de servicios de seguridad & 42 & $88 \%$ & 6 & $13 \%$ & 48 & $2 \%$ \\
\hline Operarios & 389 & $88 \%$ & 53 & $12 \%$ & 442 & $19 \%$ \\
\hline Militares & 11 & $79 \%$ & 3 & $21 \%$ & 14 & $1 \%$ \\
\hline \multicolumn{7}{|l|}{ Sector de actividad: } \\
\hline Primario & 85 & $75 \%$ & 29 & $25 \%$ & 114 & $5 \%$ \\
\hline Industria & 192 & $83 \%$ & 38 & $17 \%$ & 230 & $10 \%$ \\
\hline Construcción & 119 & $91 \%$ & 12 & $9 \%$ & 131 & $6 \%$ \\
\hline Comercio & 230 & $59 \%$ & 162 & $41 \%$ & 392 & $16 \%$ \\
\hline Transporte y logística & 89 & $82 \%$ & 20 & $18 \%$ & 109 & $5 \%$ \\
\hline Hostelería & 82 & $52 \%$ & 77 & $48 \%$ & 159 & $7 \%$ \\
\hline Inmobiliaria, finanzas, comunicación y ciencia & 194 & $62 \%$ & 118 & $38 \%$ & 312 & $13 \%$ \\
\hline Administración Pública & 117 & $57 \%$ & 88 & $43 \%$ & 205 & $9 \%$ \\
\hline Educación, sanidad y servicios sociales & 125 & $28 \%$ & 322 & $72 \%$ & 447 & $19 \%$ \\
\hline Otros servicios & 116 & $41 \%$ & 164 & $59 \%$ & 280 & $12 \%$ \\
\hline \multicolumn{7}{|l|}{ Ingresos: } \\
\hline Menos de $1100 €$ & 163 & $48 \%$ & 176 & $52 \%$ & 339 & $14 \%$ \\
\hline De 1101 a $1800 €$ & 399 & $57 \%$ & 298 & $43 \%$ & 697 & $29 \%$ \\
\hline De 1801 a $2700 €$ & 307 & $58 \%$ & 220 & $42 \%$ & 527 & $22 \%$ \\
\hline Más de $2700 €$ & 248 & $59 \%$ & 169 & $41 \%$ & 417 & $18 \%$ \\
\hline No sabe/No contesta & 232 & $58 \%$ & 167 & $42 \%$ & 399 & $17 \%$ \\
\hline \multicolumn{7}{|l|}{ Lugar de residencia: } \\
\hline Cabecera & 637 & $61 \%$ & 412 & $9 \%$ & 1049 & $44 \%$ \\
\hline Periferia & 712 & $54 \%$ & 618 & $46 \%$ & 1330 & $56 \%$ \\
\hline \multicolumn{7}{|l|}{ Región Urbana de residencia: } \\
\hline Almería & 172 & $62 \%$ & 107 & $38 \%$ & 279 & $12 \%$ \\
\hline Bahía de Cádiz & 153 & $59 \%$ & 107 & $41 \%$ & 260 & $11 \%$ \\
\hline Campo de Gibraltar & 90 & $60 \%$ & 59 & $40 \%$ & 149 & $6 \%$ \\
\hline Córdoba & 107 & $47 \%$ & 122 & $53 \%$ & 229 & $10 \%$ \\
\hline Granada & 160 & $50 \%$ & 157 & $50 \%$ & 317 & $13 \%$ \\
\hline Huelva & 90 & $56 \%$ & 70 & $44 \%$ & 160 & $7 \%$ \\
\hline Jaén & 134 & $66 \%$ & 69 & $34 \%$ & 203 & $9 \%$ \\
\hline Málaga & 193 & $56 \%$ & 153 & $44 \%$ & 346 & $15 \%$ \\
\hline Sevilla & 250 & $57 \%$ & 186 & $43 \%$ & 436 & $18 \%$ \\
\hline Total & 1349 & $57 \%$ & 1030 & $43 \%$ & 2379 & $100 \%$ \\
\hline
\end{tabular}


El ámbito al que refiere dicha encuesta viene formado por las regiones urbanas de Andalucía (ver figura 1). Son regiones definidas con criterios heterogéneos, que aglutinan municipios incluidos en los planes de ordenación del territorio de las aglomeraciones urbanas y en los consorcios metropolitanos de transporte (Regueira 2013; IECA 2011). Desgraciadamente, la fuente no permite utilizar una delimitación más consistente, como la debida a Feria y Martínez-Bernabéu (2016).

En cuanto a la metodología empleada, utilizaremos un análisis doble para abordar los dos objetivos planteados. El primer objetivo lo abordamos mediante un análisis descriptivo, consistente en la construcción de unos índices estandarizados en base 100, que comparan la propensión de cada categoría a recorrer una determinada distancia con respecto a la propensión media del total de commuters (que será considerada 100).

Para el segundo objetivo, recurrimos a la regresión logística multinomial. En este modelo, como en el análisis anterior, recodificando la variable distancia como categórica en 5 categorías: distancias muy cortas (menos de $1 \mathrm{~km}$ ) y cortas (de 1 a $3 \mathrm{~km}$ ), propias de las mujeres (el $54 \%$ de las mujeres de la muestra se encuentran en esta categoría, frente al $43 \%$ de los hombres); distancias medias (de 3 a $8,5 \mathrm{~km}$ ), sin grandes diferencias de género (el $27 \%$ de los hombres y el $24 \%$ de las mujeres); y largas (de 8,5 a 18 $\mathrm{km}$ ) y muy largas (superiores a $18 \mathrm{~km}$ ), propias de los hombres (el $29 \%$ de los hombres y el $20 \%$ de las mujeres). Se ha tomado la decisión de categorizar de esta manera la variable distancia por considerar que los distintos tipos de desplazamiento (muy corto, corto, medio, largo y muy largo) responden a motivaciones diferentes, de manera que los coeficientes de las variables y las propias variables explicativas pueden ser diferentes para cada categoría. El modelo propuesto permite incluir estos condicionantes en el modelo, cosa que no ocurriría si hubiéramos planteado una regresión lineal con la distancia como variable dependiente.

Lo que hacen los modelos de este tipo es, tomando como referencia una categoría de la variable dependiente, decir cuán probable es que ocurra un suceso dado en lugar del que se toma como referencia. En nuestro caso, puesto que queremos conocer el efecto del género en la movilidad en relación con otras variables, tomamos las distancias medias como referencia, puesto que son las que menos diferencias entre sexos presentan.

Para seleccionar las categorías de referencia de las variables independientes hemos tomado un doble criterio. El más frecuentemente usado es tomar las categorías más numerosas, lo que hemos hecho para parte de las variables. Pero en las socioeconómicas hemos dado prioridad al criterio de que, en la medida de lo posible, exista un número parejo de hombres y mujeres en las categorías de referencia; es decir, que estén poco segregadas.

Figura 1.

Delimitación de las regiones urbanas de Andalucía en la encuesta

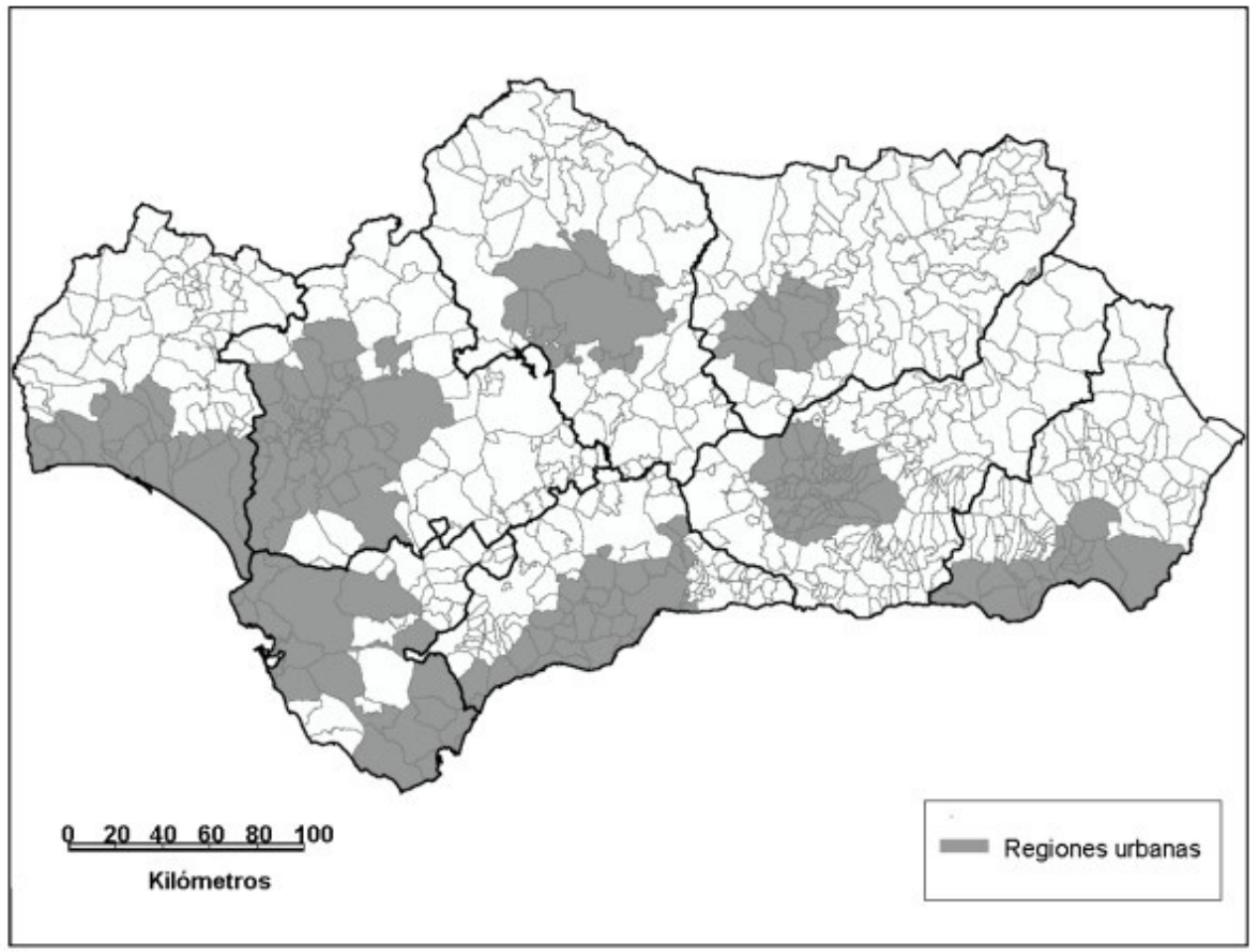

Fuente: Regueira (2013) 
En los resultados recogemos: primero, los coeficientes $(\beta)$ que indican la dirección y efecto de la variable independiente, que puede ser positivo, en caso de que incremente la probabilidad de que recorra una distancia dada $\left(p_{j}\right)$ frente a la probabilidad de que recorra un distancia media $\left(p_{3}\right)$; o negativo, en caso de que la disminuya. Segundo, el p-valor del estadístico de Wald, mediante el cual se verifica la significatividad del parámetro. Hemos considerado p-valores de 0,1 o inferiores, lo cual supone un margen de error amplio, pero adecuado al escaso volumen de datos y usualmente aceptado en ciencias sociales. $Y$ tercero, el pseudo- $R^{2}$ de Nagelkerke, que permite conocer el porcentaje de varianza explicada y contrastar la significatividad global del modelo.

El modelo asumiría la siguiente forma:

$\log \left(p_{x} / p_{3}\right)=\alpha+\beta_{\text {socio-económicas }}+\beta_{\text {socio-demográficas }}+\beta_{\text {territoriales }}$

De esta forma, establecemos en qué distancias y en qué medida el género es un factor directo (el sexo sería significativo para los modelos) o indirecto (serían significativas variables afectadas por el género, pero que presentan una pauta de movilidad propia o escasamente diferenciada por sexo); así como el peso de cada una de las características en la explicación de las distancias (a través del porcentaje de varianza explicada).

\section{RESUlTADOS: DIFERENCIAS ENTRE SEXOS EN LAS DISTANCIAS SEGÚN LAS CARACTERÍSTICAS DE LOS COMMUTERS. ¿PAUTAS DE MOVILIDAD DE GÉNERO?}

El primer paso es constatar si en Andalucía, como en otros contextos sociales y geográficos, las mujeres recorren menores distancias en sus desplazamientos por razón de trabajo. En la figura 2.a observamos que esto también ocurre en Andalucía. Las mujeres, efectivamente, trabajan más cerca de casa, ya que tienen, en conjunto, una propensión superior a la media a recorrer distancias inferiores a $3 \mathrm{~km}$, mientras los hombres tienen una propensión superior a la media a recorrer distancias superiores a los $3 \mathrm{~km}$, siendo máximas dichas diferencias en las distancias más extremas. Sin embargo, esto no debe llevarnos a pensar que tales diferencias son explicadas directamente por el género, ya que, como dijimos, puede haber otros factores que medien entre las desigualdades por sexo y la movilidad.

Figura 2.

Índices de desplazamientos por sexo y distancias recorridas para distintas categorías de población ocupada (en base cien)

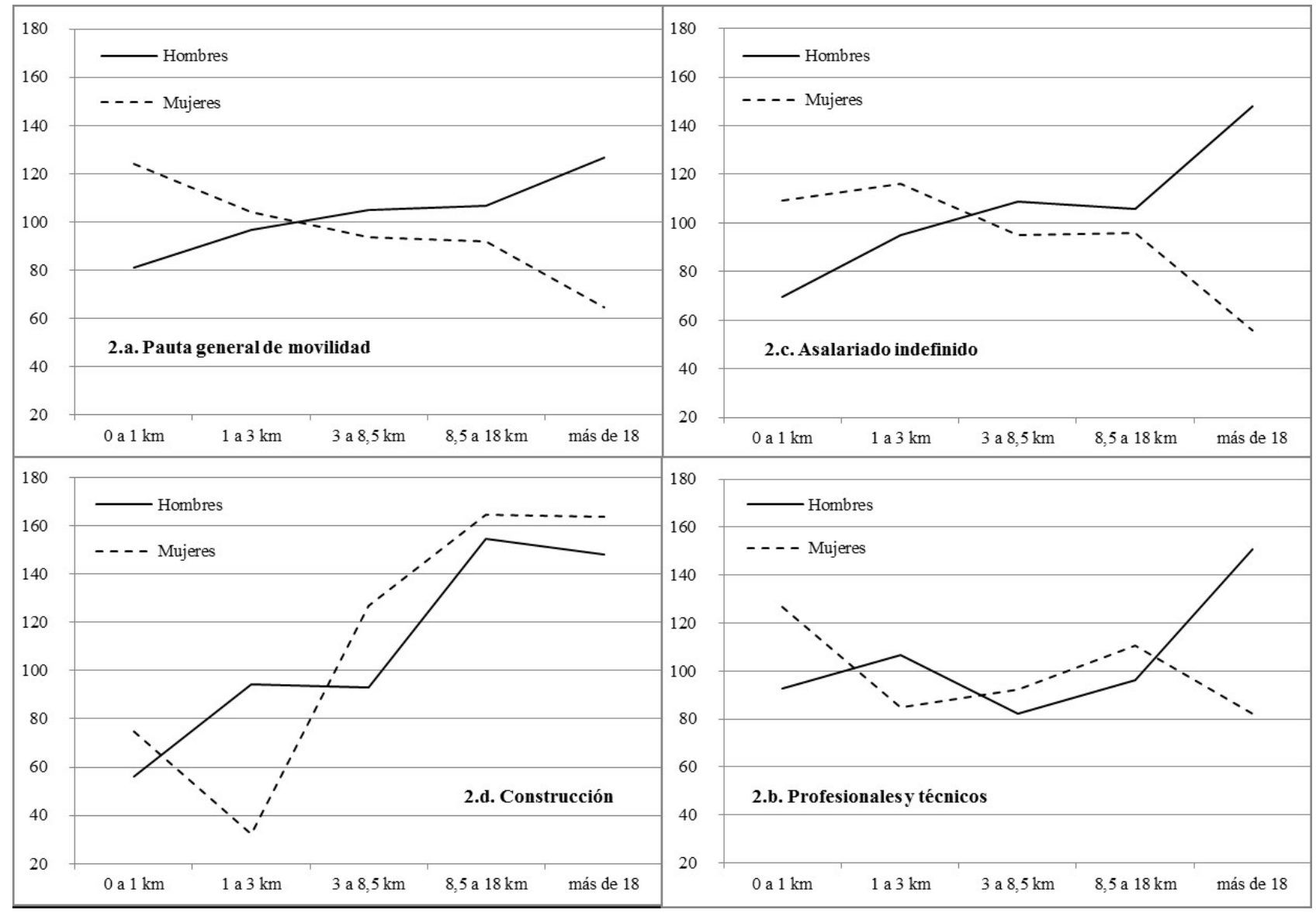

Fuente: Encuesta de Movilidad en las Regiones Urbanas de Andalucía, bloque II. Instituto de Estadística y Cartografía de Andalucía (IECA). Elaboración propia. 
Por ello, se hace necesario analizar qué categorías de ocupados tienen pautas de movilidad diferenciadas por sexos, lo que nos servirá para interpretar correctamente los resultados del posterior modelo de regresión. Se espera que las características socioeconómicas de los ocupados determinen en mayor medida las pautas de movilidad laboral (Feria y Susino 2005). En la figura 2 se presentan, además de la pauta general, tres tipos de categorías, según las diferencias entre pautas de movilidad por distancias recorridas por hombres y mujeres.

En primer lugar tenemos las categorías que tienen el mismo comportamiento que la pauta general agregada, es decir, aquellas en que las mujeres tienen una propensión superior a la media en las distancias inferiores a $3 \mathrm{~km}$, mientras en los hombres es superior a partir de dicha distancia. La distribución por sexo de los asalariados indefinidos es un ejemplo de este grupo de categorías (figura 2.b). En ellas, por su similitud con la pauta agregada, podemos decir que el género actúa directamente en la movilidad.

En segundo lugar, vemos las categorías con pautas de movilidad propias, aquellas cuya distribución por sexo presenta valores muy similares para hombres y mujeres, no ajustándose a la pauta general agregada. En la figura 2.c viene representada por los trabajadores de la construcción, caso paradigmático de este grupo. Las categorías encuadradas en este tipo son candidatos privilegiados para actuar como factores mediadores entre el género y la movilidad.

En tercer lugar, distinguimos categorías con pautas de movilidad que, pese a tener una marcada diferencia con respecto a la pauta por sexo agregada, mantienen ciertas diferencias por sexo en alguna de las distancias, especialmente en las extremas. Los técnicos y profesionales constituyen un buen ejemplo (figura 2.d). Este tipo de categorías serían mixtas, presentando a la vez un efecto directo e indirecto del género.

En el cuadro 2 se resumen las categorías de las variables independientes en tres grupos, según la distribución por sexo que presentan. De estas variables, las más importantes son las socioeconómicas, referentes a la ocupación, ya que al tratarse de movilidad laboral serán éstas, por lógica, las que condicionen en mayor medida la distancia recorrida. Sin embargo, como podemos observar, sólo unas pocas categorías tienen una marcada distribución diferencial por sexo, lo que las convierte en candidatas para actuar como factor intermedio entre género y movilidad.

Para comprobar esta idea intuitiva hemos calculado en qué ocupaciones, actividades y tipos de contratos se concentran en mayor medida las mujeres, a través de la diferencia entre el porcentaje de hom-

Cuadro 2.

Clasificación de las categorías de ocupados según su pauta de movilidad

\begin{tabular}{|l|l|l|l|}
\hline \multirow{2}{*}{ Variable } & \multicolumn{3}{|c|}{ Pauta de movilidad de cada categoría } \\
\cline { 2 - 4 } & Diferencial por sexo & Propia de la categoría & $\begin{array}{l}\text { Parcialmente diferencial } \\
\text { por sexo }\end{array}$ \\
\hline Edad & De 31 a 50 & Menor de 30 & Mayor de 50 \\
\hline Nacionalidad & Extranjero, Español & Ninguna & Ninguna \\
\hline Estado civil & Casado & Ninguna & Soltero, Estuvo casado \\
\hline Estudios & $\begin{array}{l}\text { Primarios, Secundarios, Bachi- } \\
\text { Ilerato/Formación profesional }\end{array}$ & Ninguna & Sin estudios, Superiores \\
\hline Situación profesional & Asalariado indefinido & $\begin{array}{l}\text { Otras, Empresarios y } \\
\text { autónomos }\end{array}$ & Asalariado temporal \\
\hline Tipo de contrato & Ninguna & Tiempo parcial & Tiempo completo \\
\hline Ocupación & Ninguna & Ninguna & Todas \\
\hline Rama de actividad & Comercio, Administración & $\begin{array}{l}\text { Sector primario, Hostelería, } \\
\text { Transporte y logística, } \\
\text { Construcción }\end{array}$ & Resto de categorías \\
\hline Ingresos & Pública & Ninguna & Ninguna \\
\hline Lugar de residencia & Todas & Todas & Ninguna \\
\hline Región urbana de residencia & Ninguna & Centro, Periferia \\
\hline
\end{tabular}

Fuente: Elaboración propia. 
bres y mujeres en cada categoría. Así, la mayoría de las mujeres se concentran en determinadas ocupaciones (el $75 \%$ son profesionales y técnicos en salud y educación, empleadas de oficina y trabajadoras de los servicios de cara al público), en algunas actividades (el $65 \%$ en actividades inmobiliarias, financieras, de comunicación y científicas, educación, sanidad y servicios sociales, hostelería y otras actividades de los servicios) y en tipos de contrato a tiempo parcial (más del $70 \%$ ). En la figura 3 se representan los índices de desplazamiento por distancias y sexos para las ocupaciones clasificadas según su mayor presencia de hombres o mujeres (3.a), las ramas de actividad (3.b) y dos tipos de contrato con desigual presencia de hombres y mujeres (3.c).

Si observamos la figura 3 , se aprecia que aquellos grupos en los que se concentran las mujeres (las ocupaciones y sectores con mayor presencia de mujeres y los contratos a tiempo parcial) presentan una propensión alta al desplazamiento en distancias más cortas y otra baja en las más largas.

Las mujeres se concentran en una serie de ocupaciones, actividades y tipos de contratos que, por sí mis- mas, tienen pautas de movilidad propias, semejantes para hombres y mujeres (excepto, en algún caso, en las distancias extremas). Esas ocupaciones, actividades y clases de contrato con mayor presencia de mujeres tienen un comportamiento que las hace estar, para ambos sexos, por encima de la propensión media en distancias cortas y por debajo en distancias largas.

Por tanto, de estas premisas se deriva que no sería tanto el hecho de ser mujer u hombre lo que determina la distancia recorrida, sino el tipo de ocupación, actividad o contratación (Feria y Susino 2005), siendo el género un factor explicativo de la concentración (o segregación) de hombres y mujeres en ocupaciones, actividades y tipos de contrato distintos, pero no tanto de las pautas de movilidad por sí mismas (Hanson y Pratt 1994; Hanson y Johnston 1985). Aunque esta afirmación debe ser matizada, ya que, si bien es cierto que las características socioeconómicas son factores intermedios que explican la relación entre género y movilidad, también lo es que en algunas de las ocupaciones y actividades con mayor presencia de mujeres persiste cierto efecto directo de género en las distancias extremas.

Figura 3.

Pautas espaciales de movilidad según la presencia de mujeres y hombres en ocupaciones, actividades y contratos (índices sobre la media en base cien)

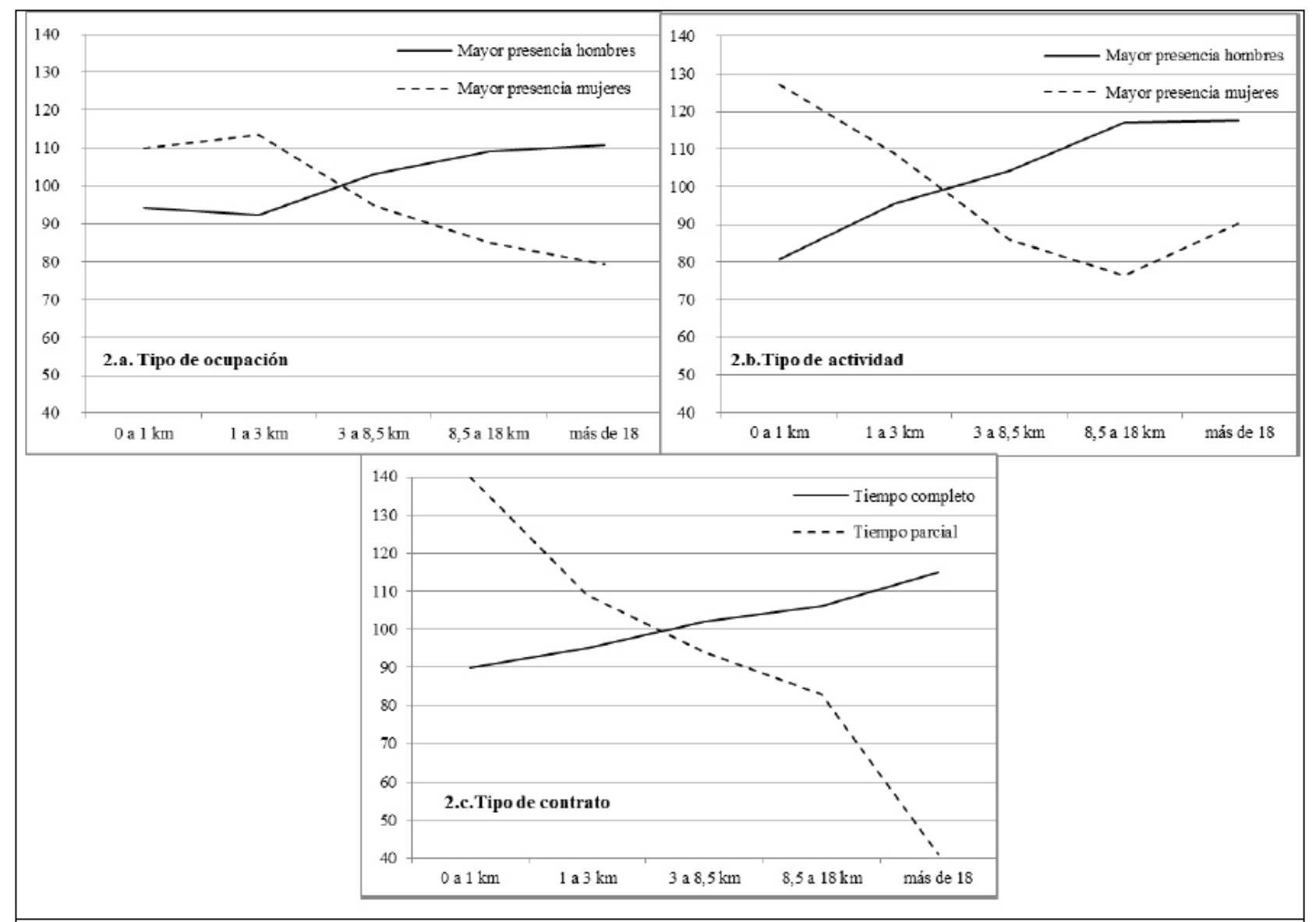

Fuente: Encuesta de Movilidad en las Regiones Urbanas de Andalucía, bloque II. Instituto de Estadística y Cartografía de Andalucía (IECA). Elaboración propia. 


\section{Resultados: el EFECTO DEL GÉNERO EN EL COMMUTING, ¿INCIDENCIA DIRECTA O INDIRECTA?}

Por todo ello, es necesario dar un paso más allá y abordar un análisis de los determinantes de commuting. Para esto utilizaremos la regresión logística multinomial, analizando el efecto diferencial, entre los sexos, de las variables ocupacionales candidatas a actuar como factores intermedios (las categorías más segregadas por sexo) y el resto de variables contempladas en los datos disponibles.
Hecho lo cual, podremos contemplar cuánto aportan el sexo y los tres bloques de variables independientes (socioeconómicas, sociodemográficas y territoriales) al porcentaje total de varianza explicada, cuantificando su efecto en cada distancia recorrida.

Para conocer la dirección y el efecto de las variables contempladas, mostramos un resumen de los resultados en el cuadro 3. Para una mayor claridad realizaremos un comentario separado de cada una de las distancias (categorías de la variable dependiente) consideradas.

Cuadro 3.

Modelo de regresión logística multinomial para explicar las distancias recorridas

\begin{tabular}{|c|c|c|c|c|c|c|c|c|}
\hline & \multicolumn{2}{|c|}{ Menos de 1 km } & \multicolumn{2}{|c|}{ De 1 a 3 km } & \multicolumn{2}{|c|}{ de 8,5 a $18 \mathrm{~km}$} & \multicolumn{2}{|c|}{ Más de 18 km } \\
\hline & $B$ & Sig. & $B$ & Sig. & $B$ & Sig. & $B$ & Sig. \\
\hline \multicolumn{9}{|l|}{ Sexo (ref=Hombre) } \\
\hline Mujer & 0,312 & $* *$ & 0,039 & - & 0,169 & - & $-0,52$ & $* * *$ \\
\hline \multicolumn{9}{|l|}{ Edad } \\
\hline Edad & $-0,13$ & $* * *$ & $-0,07$ & - & $-0,02$ & - & 0,049 & - \\
\hline Edad al cuadrado & 0,002 & $* * \star$ & 0,001 & - & 0 & - & -0 & - \\
\hline \multicolumn{9}{|l|}{ Nacionalidad (ref=Español) } \\
\hline Extranjero & $-0,32$ & - & $-0,69$ & ** & $-0,66$ & * & 0,228 & - \\
\hline \multicolumn{9}{|l|}{ Estado civil (ref=Casado) } \\
\hline Soltero & $-0,28$ & * & $-0,3$ & * & $-0,16$ & - & $-0,3$ & - \\
\hline Estuvo casado & $-0,56$ & $* *$ & 0,135 & - & $-0,09$ & - & $-0,29$ & - \\
\hline \multicolumn{9}{|l|}{ Estudios (ref=Secundaria) } \\
\hline Sin estudios & 0,134 & - & 0,12 & - & $-1,03$ & * & $-1,05$ & - \\
\hline Primarios & $-0,42$ & - & 0,005 & - & $-0,32$ & - & 0,449 & - \\
\hline Bachillerato / Formación profesional & $-0,26$ & - & $-0,25$ & - & $-0,19$ & - & 0,324 & - \\
\hline Superiores & 0,24 & - & $-0,2$ & - & 0,136 & - & 0,314 & - \\
\hline \multicolumn{9}{|l|}{ Situación profesional (ref=Asalariado indefinido) } \\
\hline Empresarios y autónomos & 0,613 & $* * *$ & 0,258 & - & 0,089 & - & $-0,08$ & - \\
\hline Asalariado temporal & 0,097 & - & $-0,13$ & - & 0,133 & - & 0,177 & - \\
\hline Otra & 0,027 & - & $-0,72$ & ** & $-0,55$ & - & $-0,44$ & - \\
\hline \multicolumn{9}{|l|}{ Tipo de contrato (ref=Tiempo completo) } \\
\hline Tiempo parcial & 0,121 & - & 0,186 & - & $-0,05$ & - & $-0,56$ & * \\
\hline \multicolumn{9}{|l|}{ Ocupación (ref=Empleados de oficina) } \\
\hline Directivos & 0,483 & - & 0,109 & - & $-0,15$ & - & 0,479 & - \\
\hline Profesionales y técnicos de salud y enseñanza & 0,435 & - & 0,405 & - & 0,216 & - & 0,497 & - \\
\hline Resto de profesionales y técnicos & 0,201 & - & $-0,04$ & - & 0,171 & - & 0,539 & * \\
\hline Trabajadores de servicios de cara al público & 0,761 & $* *$ & 0,328 & - & $-0,06$ & - & $-0,27$ & * \\
\hline Trabajadores de servicios de seguridad & $-0,27$ & - & $-0,56$ & - & 0,37 & - & 0,134 & - \\
\hline Operarios & 0,311 & - & 0,091 & - & 0,033 & - & 0,125 & - \\
\hline Militares & $-0,69$ & - & $-2,19$ & * & $-1,41$ & - & $-0,47$ & - \\
\hline \multicolumn{9}{|l|}{ Sector de actividad (ref=Hostelería) } \\
\hline Primario & 0,389 & - & $-0,36$ & - & 0,895 & * & 0,066 & - \\
\hline Industria & $-0,44$ & - & $-0,13$ & - & 0,691 & * & 0,102 & - \\
\hline Construcción & 0,262 & - & 0,504 & - & 1,497 & $* * *$ & 0,986 & * \\
\hline Comercio & 0,117 & - & 0,16 & - & 0,698 & * & 0,75 & - \\
\hline Transporte y logística & $-1,02$ & $* *$ & $-0,07$ & - & 0,347 & - & 0,401 & - \\
\hline
\end{tabular}




\begin{tabular}{|c|c|c|c|c|c|c|c|c|}
\hline Inmobiliaria, finanzas, comunicación y ciencia & 0,736 & ** & 0,898 & $* * *$ & 0,606 & - & 0,743 & - \\
\hline Administración Pública & 1,278 & *** & 1,021 & $* * *$ & 0,861 & ** & 0,923 & * \\
\hline Educación, sanidad y servicios sociales & 0,713 & ** & 0,53 & * & 0,323 & - & 0,929 & * \\
\hline Otros servicios & 0,852 & *** & 0,531 & * & $-1,24$ & *** & 0,793 & - \\
\hline \multicolumn{9}{|l|}{ Ingresos (ref=Más de $2700 €$ ) } \\
\hline Menos de $1100 €$ & 1 & *** & 0,499 & ** & 0,073 & - & $-0,23$ & - \\
\hline De 1101 a $1800 €$ & 0,548 & ** & $-0,05$ & - & $-0,46$ & ** & $-0,37$ & - \\
\hline De 1801 a $2700 €$ & 0,618 & *** & 0,209 & - & 0,154 & - & $-0,09$ & - \\
\hline No sabe/no contesta & 0,907 & *** & 0,323 & - & $-0,21$ & - & 0,007 & - \\
\hline \multicolumn{9}{|l|}{ Lugar de residencia (ref=Cabecera de la región) } \\
\hline Periferia & $-0,28$ & ** & 0,222 & * & $-0,92$ & $* * *$ & $-0,8$ & *** \\
\hline \multicolumn{9}{|l|}{ Región de residencia (ref=Sevilla) } \\
\hline Almería & 0,138 & - & 0,323 & - & 0,305 & - & 0,175 & - \\
\hline Cádiz & 0,409 & * & 0,697 & $* * *$ & 0,123 & - & 0,493 & - \\
\hline Algeciras & 0,631 & ** & 0,393 & - & 0,24 & - & 0,164 & - \\
\hline Córdoba & 0,58 & $* *$ & 0,714 & $* * *$ & 0,014 & - & 0,761 & $* *$ \\
\hline Granada & 0,373 & * & $-0,03$ & - & 0,102 & - & $-0,23$ & - \\
\hline Huelva & 1,396 & $* * *$ & 0,928 & $* * *$ & 0,943 & $* * *$ & 1,105 & *** \\
\hline Jaén & 2,008 & $* * *$ & 1,671 & $* * *$ & 1,381 & $* * *$ & 1,527 & $* * *$ \\
\hline Málaga & 0,075 & - & 0,27 & - & 0,941 & $* * *$ & 0,681 & $* *$ \\
\hline Intersección & 0,262 & - & 0,37 & - & $-0,81$ & - & $-2,18$ & - \\
\hline
\end{tabular}

${ }^{*}$ p-valor $<0,1 ;{ }^{* *}$-valor $<0,05 ;{ }^{* * *}$ p-valor $<0,01$

Fuente: Encuesta de Movilidad en las Regiones Urbanas de Andalucía, bloque II. Instituto de Estadística y Cartografía de Andalucía (IECA). Elaboración propia.

En las distancias de proximidad, las inferiores a $1 \mathrm{ki}-$ lómetro, el análisis muestra que la incidencia del género es directa, al aparecer la variable sexo como significativa y con signo positivo, ratificándose la mayor propensión de las mujeres a trabajar cerca de su residencia.

Aparte del sexo, es destacable que solo en estas distancias aparecen variables sociodemográficas significativas, concretamente dos. De un lado el estado civil, que muestra, dado el signo negativo de solteros y de los que estuvieron casados, que las personas casadas, independientemente del sexo, tienen una mayor propensión a trabajar más cerca de casa. De otro la edad, que aparece con signo negativo, lo que indica que cuanto más joven se es, mayor probabilidad se tiene de trabajar cerca de casa. No obstante, el hecho de que su cuadrado aparezca como significativo y con signo positivo indica que la edad puede vincularse de forma no lineal con la distancia, siendo mayor la relación en las edades más elevadas.

Respecto de las variables socioeconómicas, encontramos variaciones significativas en tres de las contempladas. La situación profesional muestra la mayor propensión de los empresarios y autónomos a trabajar a distancias de proximidad; algo lógico si tenemos en cuenta que la mayoría de los sujetos encuadrables bajo esta categoría son clases medias patrimoniales, cuyos negocios se encuentran frecuentemente en su propio domicilio o muy cerca del mismo. En cuanto a la ocupación y la actividad, vemos que los trabajadores de los servicios cara al público, los que trabajan en educación, sanidad y servicios sociales, otros servicios, actividades científicas, inmobiliarias, financieras y de comunicación y en la Administración Pública, tienen una propensión significativa y positiva a trabajar en estas distancias. Puesto que las tres primeras categorías mencionadas tienen gran presencia de mujeres, esto muestra que el efecto del género en la explicación de estas distancias es también indirecto, algo que se refuerza al aparecer con signo negativo una categoría con preponderancia de hombres, como es el transporte y la logística. En cuanto a los ingresos, vemos que todas las categorías presentan una propensión significativa y positiva respecto de la categoría de referencia (más de $2700 €$ los que más ganan), lo que apunta a una relación positiva entre salarios y distancia: a más distancia, más salarios y viceversa.

En lo que respecta a las variables territoriales, el signo negativo asociado al hecho de vivir en cabeceras es difícil de explicar; quizás se deba a que la categoría de referencia con la que se compara (las periferias) no sólo incluye municipios metropolitanos, sino también municipios menos urbanos donde predominan desplazamientos de proximidad. En cuanto a las regiones urbanas de residencia, vemos que, en general, todas presentan una mayor probabilidad de realizar movimientos de proximidad que la de referencia (Sevilla) excepto Málaga, algo explicable por la gran extensión de éstas dos áreas. 
Si pasamos a las distancias de 1 a $3 \mathrm{~km}$ (las cortas) vemos que el sexo no aparece como variable significativa, pudiéndose afirmar que el género no influye directamente en la probabilidad de recorrerlas. Sin embargo, sí que incide indirectamente. Así, las categorías sectoriales que aparecían en las distancias inferiores a 1 kilómetro (los servicios cara al público, los que trabajan en educación, sanidad y servicios sociales, otros servicios, actividades científicas, inmobiliarias, financieras y de comunicación y en la Administración Pública) vuelven a aparecer con signo positivo, lo que indica de nuevo su mayor propensión a recorrer estas distancias frente a las medias. También se observan variaciones significativas en los ingresos, de manera que, como era de esperar, los que menos ganan (menos de $1100 €$ al mes) tiene una propensión superior a recorrer distancias cortas. En las variables territoriales se aprecia que el hecho de vivir en las regiones urbanas de Cádiz, Huelva, Córdoba o Jaén incrementa la propensión a recorrer distancias cortas en lugar de distancias medias, un efecto similar al que veíamos en las distancias muy cortas.

Las distancias largas, al igual que las cortas, no presentan ningún efecto directo de género. Pero sí indirecto, al aparecer actividades como la construcción, la industria y el sector primario (con predominio de hombres) con signo positivo, mientras el resto de actividades de servicios (en las que predominan las mujeres) aparece con signo negativo.

Respecto a estas distancias merece la pena comentar las variables territoriales. De un lado, vemos que el hecho de vivir en cabeceras hace que la probabilidad de recorrer estas distancias sea inferior a la probabilidad de recorrer distancias medias, lo cual es bastante lógico si pensamos que la mayoría de empleos se concentran en los municipios centrales de las áreas metropolitanas, y muchos de los municipios periféricos son dependientes de las cabeceras que articulan sus respectivas áreas metropolitanas. De otro, se aprecia que residir en algunas regiones urbanas, como Huelva, Jaén o Málaga, incrementa esta probabilidad, lo que puede explicarse por diferentes razones. En los casos de Huelva y Jaén se debe a que sus regiones urbanas (tal y como son definidas en la encuesta analizada) comprenden municipios lejanos, con fuerte centralidad en el empleo: por ejemplo, Andújar en Jaén o Lepe en Huelva. Por otro lado, el caso de Jaén también es explicable porque el fenómeno metropolitano es aún incipiente $y$, en estas fases de desarrollo, se produce una transición de las economías rurales a las urbanas, generándose una dependencia del emergente centro metropolitano que lleva a los habitantes de esas zonas distantes y en transición a desplazarse largas distancias hacia el centro metropolitano. El caso malagueño puede explicarse por la particularidad de su desarrollo y configuración metropolitana en torno a dos núcleos centrales (Málaga y Marbella) y a un conjunto de municipios que hacen de charnela entre ambos, como Mijas o Fuengirola.

Finalmente, en las distancias muy largas de nuevo aparece el sexo como variable explicativa directa de la distancia, teniendo las mujeres una probabilidad inferior a los hombres de realizar estos desplazamientos.

Pero también se aprecia una incidencia del género indirecta, al aparecer con signo negativo los trabajadores de servicios de cara al público, una categoría con gran predominio de mujeres; así como categorías con mucha presencia de hombres, como la construcción, con signo positivo. No obstante, también vemos que son significativas otras categorías, como el resto de profesionales y técnicos y los que trabajan en sanidad, educación y servicios sociales y en la Administración Pública. Si bien los primeros son más difíciles de interpretar, las dos últimas categorías pueden explicarse por el hecho de que las plazas públicas, a las que mayoritariamente se accede por oposición o interinidad, están sujetas a cambios y traslados dentro de la región que ocurren normalmente tras fijar el domicilio.

Por otro lado, el resto de variables significativas en el modelo para estas distancias son territoriales. Primero, el hecho de vivir en las cabeceras metropolitanas, cuya explicación tratamos más arriba. $Y$ segundo, otra vez las regiones urbanas de Málaga, Huelva, Jaén y Córdoba. Como decíamos antes, la presencia en estas regiones de otros municipios con centralidad (Fuengirola, Lepe, Andújar, Linares, Lucena...), el escaso grado de desarrollo metropolitano de Córdoba y Jaén y la particular configuración metropolitana policéntrica de Málaga, pueden explicar esta tendencia.

A lo largo del comentario de cada una de las distancias hemos podido ver que la variable sexo sólo aparece como significativa en las distancias más extremas, pudiéndose afirmar que el género actúa directamente sólo en estas distancias.

Por otro lado, se deduce del modelo que el género también actúa como variable indirecta. Lo veíamos cuando mostrábamos que las profesiones con mayor presencia femenina aparecían con signo positivo en las distancias cortas y muy cortas, mientras algunas categorías predominantemente masculinas aparecían con signo positivo en las distancias superiores largas y muy largas. Por tanto, el género puede estar actuando de manera encubierta a través de categorías socioeconómicas con altos niveles segregación por sexo. Por último, el efecto de las variables territoriales es considerable, especialmente el hecho de vivir en las cabeceras metropolitanas y la región urbana de residencia concreta. Menor incidencia, aunque nada desdeñable, presentan el nivel de estudios, la edad o los ingresos. En concreto, los valores de este último parecen indicar que, a mayor ingreso, mayor distancia, coincidiendo con algunos estudios 
internacionales (Clark y Wang 2005), o con el conocido como modelo urbano estándar (Simpson 1992; Alonso 1964), que afirma que la principal motivación para el commuting reside, precisamente, en los mayores salarios.

En total, con todas las variables incluidas en el modelo, se consigue una $R$ cuadrado de Nagelkerke de 0,237 , lo cual indica que se alcanza a explicar un $23,7 \%$ de la varianza total, lo que no está nada mal para un modelo de estas características.

Tanto el modelo como el análisis descriptivo parecen indicar que las diferencias de sexo en la movilidad son explicables a través del género, pero no tanto de forma directa como indirectamente, a través de factores intermedios de carácter socioeconómico. Para cuantificar de alguna forma este efecto del género y el resto de variables, hemos analizado el cambio en el coeficiente de ajuste pseudo- $R^{2}$ que producen el sexo y otras variables relevantes.

En el cuadro 4 podemos apreciar que el sexo apenas aporta un $0,6 \%$ al total de varianza explicada por el modelo, frente al $5 \%$ de las categorías de ocupación y actividad, las cuales (como vimos en el descriptivo) presentan, en general, índices altos de segregación por sexo. Esto nos lleva a afirmar con mayor rotundidad que el efecto directo del género en la movilidad es cuantitativamente menor que el efecto indirecto que se produce a través de la segregación ocupacional y sectorial.

\section{Conclusiones}

Las diferencias de sexo en la movilidad son, en efecto, producto de las desigualdades de género. Sin embargo, la mera constatación de diferencias de sexo no supone más que un acercamiento descriptivo que deja por resolver la cuestión principal, que consiste en conocer cómo actúan dichas desigualdades. Así, cualquier estudio que pretenda orientar políticas de igualdad de género o, simplemente, ahondar en un conocimiento científico más sistemático de la cuestión, no debe quedarse en este primer nivel descriptivo, que sin duda es un paso previo ineludible, sino que debe transcenderlo, acercándose a los factores a través de los cuales dichas desigualdades operan. Precisamente esta fue la reflexión que nos llevó a realizar el presente trabajo y a formular nuestro interrogante de partida: ¿cómo operan las desigualdades de género en el commuting?

Si bien investigaciones precedentes se centran en constatar y comprender la naturaleza de la incidencia directa a través de la hipótesis del enclaustramiento femenino (Rapino y Cook 2011; Rapino 2008; Crane 2007; Cristaldi 2005), o indirecta a través de lo que Rosenbloom y Burns (1993) llamaron teoría económica tradicional (MacDonald 1999; Gordon, Kumar y Richardson 1989), nosotros decidimos acotar nuestros objetivos de forma más concreta y simple, pero que supone nuestra principal aportación a los estudios sobre género y commuting: tratar de cuantificar el valor explicativo de las distintas hipótesis.

Partiendo de este objetivo general y con los resultados obtenidos podemos afirmar que, para el caso de Andalucía, el marco explicativo de la teoría tradicional es capaz de dar cuenta de manera más satisfactoria de las diferencias por sexo en la movilidad, tal como Feria y Susino (2005) sugirieron, sin que ello suponga rechazar explicaciones complementarias desde la teoría del enclaustramiento, que también tienen su margen de potencial explicativo (Olmo y Maeso 2013).

Sin embargo, la particularidad del caso analizado sugiere nuevas preguntas: ¿operará de la misma manera el género en el commuting en otros contextos sociales y geográficos? Y ¿hasta qué punto los resultados obtenidos dependen de las características de la base de datos utilizada?

Cuadro 4.

Cambio en pseudo-R cuadrado al eliminar las variables más relevantes para el modelo, frente al pseudo- $R$ cuadrado total obtenido.

\begin{tabular}{|l|c|c|}
\hline Eliminadas & $\mathbf{R}^{\mathbf{2}}$ & ${\text { Cambio en } \mathbf{R}^{\mathbf{2}}}^{0,60 \%}$ \\
\hline Sexo & $23,10 \%$ & $0,10 \%$ \\
\hline Tipo de contrato & $23,60 \%$ & $0,90 \%$ \\
\hline Situación profesional & $22,80 \%$ & $1,30 \%$ \\
\hline Ocupación & $22,40 \%$ & $3,69 \%$ \\
\hline Actividad & $20,01 \%$ & $1,50 \%$ \\
\hline Ingresos & $22,20 \%$ & $2,70 \%$ \\
\hline Región urbana & $21,00 \%$ & $4,90 \%$ \\
\hline Lugar de residencia & $18,80 \%$ & $0,00 \%$ \\
\hline Ninguna & $23,70 \%$ & \\
\hline
\end{tabular}

Fuente: Encuesta de Movilidad en las Regiones Urbanas de Andalucía, bloque II. Instituto de Estadística y Cartografía de Andalucía (IECA). Elaboración propia. 
Sin duda, las regiones urbanas andaluzas constituyen un contexto particular en comparación con otros ámbitos en los que anteriormente se ha estudiado esta cuestión. Por tanto, lo que sea cierto para Andalucía puede no serlo para otras realidades urbanas. Sólo la investigación comparativa internacional podrá contestar a esta cuestión en el futuro. En todo caso parece confirmarse lo que otros estudios ya han señalado: que las diferencias en la movilidad por razón de trabajo entre mujeres y hombres están determinadas por el género, tanto directa como, sobre todo, indirectamente. No obstante, hay factores que se sitúan más allá del género, puesto que pueden afectar a ambos sexos por igual, como son los territoriales.

Analizar el caso andaluz tiene sentido, ya que la encuesta utilizada en este trabajo, debida al Instituto de Estadística y Cartografía de Andalucía, es una fuente rigurosa e inusual; más aún en nuestro contexto español, donde apenas existen encuestas específicas sobre movilidad cotidiana. No obstante, no está exenta de dificultades.

Esta fuente parte de una delimitación de las regiones urbanas andaluzas que no sigue criterios homogéneos para todas ellas. Junto a delimitaciones más próximas a las áreas metropolitanas que se articulan en torno a Sevilla o Granada, por ejemplo, otras claramente sobrepasan los límites de tales áreas, como en los casos de Huelva o Jaén. Por eso debemos preguntarnos si la importancia que en los modelos utilizados tienen las variables territoriales, se debe a diferencias reales entre las pautas de movilidad de los ocupados en cada una de esas regiones urbanas concretas, o es provocada por los diferentes criterios seguidos en su delimitación. Sin salir de Andalucía, se hace necesario, por tanto, intentar en el futuro partir de delimitaciones de áreas definidas con criterios homogéneos (Feria y Martínez-Bernabéu 2016). Pero eso nos obligaría a recurrir a otras fuentes, como las censales, utilizadas en otro trabajo anterior (Feria y Susino 2005).

Es necesario, por tanto, profundizar en esta línea de investigación. Primero, analizando otras áreas, ampliando el ámbito al conjunto de las áreas metropoli- tanas españolas, pues es en las zonas intensamente urbanizadas donde puede apreciarse mejor la articulación entre desplazamientos, territorio, ocupación y género. Segundo, utilizando fuentes que permitan el uso de delimitaciones más rigurosas de tales áreas, realizadas con los mismos criterios para todas ellas. Tercero, preguntándonos, más allá de los factores intervinientes en las diferencias de movilidad entre ambos sexos, por los mecanismos que subyacen a las desigualdades de género que aquellas traslucen. Es decir, no solo cómo opera el enclaustramiento para que las mujeres tengan mayor presencia en las distancias más cortas o menor en las más largas, sino también cómo llega a producirse la segregación entre hombres y mujeres en muchas profesiones o ramas de actividad y que, indirectamente, provocan las diferencias en las distancias recorridas por ambos sexos. En este sentido, deberíamos contestar a una alternativa crucial: ¿trabajan las mujeres en determinadas ocupaciones y sectores porque ello les permite recorrer distancias menores? $\mathrm{O}$, al contrario, ¿sus desplazamientos son más cortos porque eso caracteriza a las ocupaciones o sectores en los que las mujeres tienen mayor presencia? En ambos casos el género explicaría, indirectamente, las diferencias en el commuting, pero según lógicas diferentes. En el primer caso, como una especie de extensión de las teorías del enclaustramiento, lo que, de confirmarse, las reforzaría. En el segundo, como un efecto más de desigualdades que operan a otro nivel. Pero confirmar tales hipótesis requiere otras fuentes y otro diseño de investigación.

\section{Agradecimientos}

Este trabajo es fruto del proyecto coordinado de investigación Movilidad y ciudad real: dinámicas y cambios territoriales y sociales en España (CSO201455780-C3), financiado por el Ministerio de Economía y Competitividad en el marco del Programa Estatal de Fomento de la Investigación Científica y Técnica de Excelencia, Subprograma Estatal de Generación de Conocimiento, convocatoria 2014, modalidad Proyectos de I+D.

\section{ReferenCIAS BibLIOGRÁficas}

Alonso, W. 1964. Location and land use. Cambridge, Massachussets: Harvard University Press.

Artís, M., J. Romaní y J. Suriñach. 2000. "Determinants of Individual Commuting in Catalonia, 1986-91: Theory and Empirical Evidence". Urban Studies 37: 14311450. https://doi.org/10.1080/00420980020080191

Casado, J.M. 2000. "Diferencias de género en los desplazamientos cotidianos por razones laborales". Documentos de trabajo del IVIE 2000-03. Instituto Valenciano De Investigaciones Económicas, Universidad de Alicante. Alicante.
Cheshire, P. 1995. "A new phase of urban development in Western Europe? The evidence for the 1980s". Urban Studies 32: 1045-1063. https://doi. org/10.1080/00420989550012564

Clark, W. A. V., y W. W. Wang. 2005. "Job access and commute penalties: balancing work and residence in Los Angeles". Urban Geography 26: 610-626. https://doi. org/10.2747/0272-3638.26.7.610

Crane, R. 2007. "Is there a quite Revolution in Women's Travel? Revisiting the Gender Gap in Commuting". Journal of the American Planning Association 73: 298316. https://doi.org/10.1080/01944360708977979 
Cristaldi, F. 2005. "Commuting and Gender in Italy: AMethodological Issue". The Professional Geographer 57: 268-284. https:// doi.org/10.1111/j.0033-0124.2005.00477.x

Dargay, J.M. y S. Clark. 2012. "The determinants of long distance travel in Great Britain". Transport. Research Part A 46: 576-587. https://doi.org/10.1016/j.tra.2011.11.016

Díaz-Muñoz, M.A. 1989. "Movilidad femenina en la ciudad. Notas a partir de un caso". Documents d'anàlisi geogràfica 14: 219-239.

Díaz-Muñoz, M.A. y F.J. Jiménez-Gigante. 2007. "Transportes y movilidad: ¿necesidades diferenciales según género?". Terr@ Plural 1: 91-101.

Feria, J.M. 2004. "Problemas de definición de las áreas metropolitanas en España”. Boletín de la A. G.E. 38: 85-99.

Feria, J.M. 2015. Definición y pautas generales de dinámica y organización espacial. Áreas metropolitanas andaluzas. Sevilla: Consejería de Fomento y Vivienda de la Junta de Andalucía.

Feria, J.M. y J. Susino (coords.). 2005. Movilidad por razón de trabajo en Andalucía 2001. Sevilla: Instituto de Estadística y Cartografía de Andalucía.

Feria, J.M. y L. Martínez-Bernabeu, 2016. "La definición y delimitación del sistema metropolitano español. Permanencias y cambios entre 2001 y 2011". Ciudad y Territorio. Estudios Territoriales 187: 11-26.

García-Palomares, J.C. 2008. "Incidencia en la movilidad de los principales factores de un modelo metropolitano cambiante". EURE 34(101): 5-24. https://doi. org/10.4067/S0250-71612008000100001

Gordon, P., A. Kumar y H.W. Richardson. 1989. "The spatial mismatch hypothesis: Somenewevidence". Urban Studies 26: 315-326. https://doi.org/10.1080/00420988920080321

Hanson, S. e I. Johnston. 1985. "Gender differences in work-trip length: explanations and implications". Urban geography 6: 193-219. https://doi.org/10.2747/0272-3638.6.3.193

Hanson, S., y G. Pratt. 1994. "On Suburban Pink Collar Ghettos: The Spatial Entrapment of Women? by Kim England". Annals of the Association of American Geographers 84(3): 500-502. https://doi. org/10.1111/j.1467-8306.1994.tb01873.x

Instituto de Estadística y Cartografía de Andalucía. 2011. Encuesta Social 2011: Movilidad en las Regiones Urbanas de Andalucía. Sevilla: Instituto de Estadística y Cartografía de Andalucía.

Kawase, M. 2004. "Changing gender differences in commuting in the Tokyo metropolitan suburbs". GeoJournal 61: 247-253. https://doi.org/10.1007/s10708-004-3679-2

Kim, C., S. Sang, Y. Chun y W. Lee. 2012. "Exploring urban commuting imbalance by jobs and gender". Applied Geography 32: 532-545. https://doi.org/10.1016/j.apgeog.2011.07.001

Lee, B.S. y J.F. McDonald. 2003. "Determinants of Commuting Time and Distance for Seoul Residents: The Impact of Family Status on the Commuting of Women". Urban Studies 40: 1283-1302. https://doi. org/10.1080/0042098032000084604

MacDonald, H.I. 1999. "Women's employment and commuting: explaining the links". Journal of Planning Literature 13: 267-283. https://doi.org/10.1177/08854129922092397

Madden, J.F. 1981. "Why women work closer to home?". Urban Studies 18: 181-194. https://doi. org/10.1080/00420988120080341

McKenzie, B. y M. Rapino M. 2011. Commuting in the United States: 2009. Washington D.C: American Community Survey Reports ACS-15. US Census Bureau.
McLafferty, S. y V. Preston. 1997. "Gender, race, and the determinants of commuting: New York in 1990". Urban Geography 18: 192-212. https://doi.org/10.2747/02723638.18.3.192

McQuaid, R.W. y T. Chen. 2012. "Commuting times: the role of gender, children and part-time work". Research in transportation economics 34: 66-73. https://doi. org/10.1016/j.retrec.2011.12.001

Miralles-Guasch, M. y C.M. Melo. 2012. "Las divergencias de género en las pautas de movilidad en Cataluña, según edad y tamaño del municipio". Revista LatinoAmericana de Geografia e Gênero 3: 49-60. https:// doi.org/10.5212/Rlagg.v.3.i2.049060

Moss J., C.G. Jack y M.T. Wallace. 2004. "Employment location and associated commuting patterns for individuals in disadvantaged rural areas in Northern Ireland". Reg. Studies, 38(2): 121-136. https://doi. org/10.1080/0034340042000190118

Neto, R.S., G. Duarte y A. Páez. 2014. "Gender and commuting time in Sao Paulo Metropolitana Region". Urban Studies 52: 298-313. https://doi.org/10.1177/0042098014528392

Olmo, M.I. y E. Maeso. 2013. "Diferencias de género en la movilidad en las regiones urbanas de Andalucía." Revista Latino-Americana de Geografia e Gênero 4: 13-28. https://doi.org/10.5212/Rlagg.v.4.i2.013028

Preston, V. y S. McLafferty. 2016. "Revisiting Gender, Race, and Commuting in New York". Annals of the American Association of Geographers 106: 300-310. https://doi. org/10.1080/00045608.2015.1113118

Rapino, M.A. 2008. "Gender roles and spatial entrapment". Tesis doctoral, Departamento de Geografía, Universidad de Connecticut, Connecticut, USA.

Rapino, M.A. y T.J. Cooke. 2011. "Commuting, gender roles, and entrapment: A national study utilizing spatial fixed effects and control groups". The Professional Geographer 63: 277-294. https://doi.org/10.1080/003 30124.2010.547790

Regueira, I. E. 2013. "La movilidad cotidiana en las regiones urbanas de Andalucía. Movilidad según tipo de poblamiento". Documentos de trabajo del Instituto de Estadística y Cartografía de Andalucía. Sevilla.

Rodríguez-Moya, J.M. y García-Palomares, J.C. 2012. "Diversidad de género en la movilidad cotidiana en la Comunidad de Madrid." Boletín de la Asociación de Geógrafos Españoles 58: 105-131.

Rosenbloom, S. 2006. “Understanding women's and men's travel patterns". Pp. 7-28 en Research on Women's Issues in Transportation: Report of a Conference. Editado por Transportation Research Board. Washington D.C: Transportation Research Board.

Rosenbloom, S. y E. Burns. 1993. "Gender difference in commuter travel in Tucson: Implications for travel demand management programs". Transportation Research Record 1404: 82-90.

Sandow, E. 2008. "Commuting behaviour in sparsely populated areas: evidence from northern Sweden". Journal of Transport Geography 16: 14-27. https://doi. org/10.1016/j.jtrangeo.2007.04.004

Sang, S., M. O'Kelly y M.P. Kwan. 2011. "Examining commuting patterns: Results from a journey-to-work model disaggregated by gender and occupation". Urban Studies, 48(5): 891-909. https://doi.org/10.1177/0042098010368576

Simpson, W.1992.Urban structure and the labour market. Oxford: Clarendon Press. 
JOSÉ MANUEL TORRADO RODRÍGUEZ es licenciado en Sociología por la Universidad de Granada y ha realizado el máster "Análisis Geográfico en la Ordenación del Territorio. Tecnologías de Información Geográfica" de la misma Universidad. Actualmente es personal investigador con cargo al proyecto Procesos de Reconfiguración metropolitana (CSO201455780-C3-3-P) y realiza su tesis doctoral en torno a las dinámicas de movilidad residencial y transformación de las ciudades centrales.

JAVIER ROMANÍ FERNÁNDEZ es doctor en Ciencias Económicas por la Universidad de Barcelona. Actualmente es profesor titular de universidad en el Departamento de Econometría, Estadística y E.A. de dicha Universidad e investigador del grupo AQR-IREA. Sus principales líneas de investigación se centran en la Economía Regional y Urbana, el commuting, las migraciones, el turismo y el análisis de impacto económico.

JOAQUÍN SUSINO ARBUCIAS es doctor en Sociología por la Universidad de Granada. Actualmente es profesor contratado y doctor del Departamento de Sociología de la Universidad de Granada. Ha desarrollado su actividad profesional e investigadora en los campos de la planificación urbana y territorial, la sociología urbana y la demografía, en los que ha publicado distintos trabajos. 